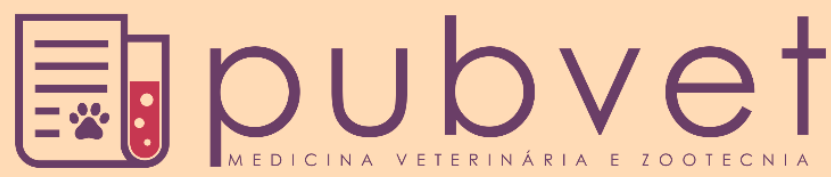

https://doi.org/10.31533/pubvet.v14n11a692.1-5

\title{
Vértebra transicional toracolombar em um felino: Relato de caso
}

\author{
Mariana Wilhelm Magnabosco ${ }^{1 *} \mathscr{9}$, Péter de Lima Wachholz ${ }^{2} \Phi$, Tiago Trindade Dias ${ }^{3 \oplus 9}$, Yohana \\ Fernanda Henz ${ }^{409}$, Alana Moraes de Borba ${ }^{19}$, Thaís Cozza dos Santos ${ }^{509}$, Tainá Evaristo Mendes \\ Cardoso $^{69}$, Leendert Kleer Neto ${ }^{50}$, Guilherme Albuquerque de Oliveira Cavalcanti ${ }^{70}$
}

${ }^{1}$ Médica Veterinária Residente em Diagnóstico por Imagem pela Universidade Federal de Pelotas. Pelotas - RS, Brasil.

${ }_{2}^{2}$ Médico Veterinário Residente em Clínica Médica de Pequenos Animais pela Universidade Federal de Pelotas. Pelotas - RS, Brasil.

${ }^{3}$ Médico Veterinário Graduado pela Universidade da Região da Campanha. Bagé - RS, Brasil.

${ }^{4}$ Médica Veterinária Graduada pela Universidade Federal de Santa Catarina. Curitibanos - SC, Brasil.

${ }^{5}$ Médico(a) Veterinária Mestranda pela Universidade Federal de Pelotas. Pelotas - RS, Brasil.

${ }^{6}$ Graduanda em Medicina Veterinária pela Universidade Federal de Pelotas. Pelotas - RS, Brasil.

${ }^{7}$ Médico Veterinário Professor adjunto da Universidade Federal de Pelotas, Departamento de Clínicas Veterinárias. Pelotas - RS, Brasil.

Autor para correspondência, E-mail: mariwmvet@gmail.com

Resumo. Vértebra transicional é uma anomalia congênita caracterizada por uma vértebra que assume características anatômicas de outra vértebra em região adjacente e ocorre nas junções cervicotorácica, toracolombar, lombossacra e sacrococcígea. É a anomalia congênita mais comum em cães e gatos, sendo diagnosticada através da radiografia. $O$ presente trabalho tem como objetivo relatar um caso de vértebra de transição toracolombar em um felino, de 11 anos, abordando sobre os achados radiográficos. A paciente foi recebida no Laboratório de Diagnóstico por Imagem e Cardiologia (LADIC) do Hospital de Clínicas Veterinárias da Universidade Federal de Pelotas para realização de radiografia torácica com o objetivo de investigar a presença de metástase pulmonar. Radiografias em incidências ortogonais de tórax foram obtidas e demonstraram a presença de costelas bilateralmente inseridas em primeira vértebra lombar (L1), além de espondiloses ao longo da coluna vertebral torácica. A paciente não possuía sinais clínicos compatíveis com essas alterações vertebrais. A vértebra transicional toracolombar possui grande importância na tomada de decisão de clínicos e cirurgiões perante procedimentos torácicos. O radiologista deve se atentar para essa alteração e comunicá-la para que clínico, cirurgião e tutor tomem conhecimento, evitando assim possíveis erros médicos que prejudiquem o paciente.

Palavras chave: anomalia congênita, coluna vertebral, gato, radiografia

\section{Thoracolumbar transitional vertebrae in a feline: Case report}

\begin{abstract}
The transitional vertebra is a congenital anomaly characterized by a vertebra that assumes anatomical characteristics of another vertebra in the adjacent region and occurs at the cervicothoracic, thoracolumbar, lumbosacral, and sacrococcygeal junctions. It is the most common congenital anomaly in dogs and cats and is diagnosed by radiography. The present work aims to report a case of thoracolumbar transitional vertebrae in a feline, 11 years old, addressing the radiographic findings. The patient was received in the Laboratório de Diagnóstico por Imagem e Cardiologia (LADIC) of the Universidade Federal de Pelotas Veterinary Teaching Hospital for thoracic radiography to investigate the presence of lung metastasis. Radiographs in orthogonal views of the thorax were obtained and demonstrated the presence of ribs bilaterally inserted in the first lumbar vertebrae (L1), in addition to spondylosis along the thoracic spine. The patient did not have clinical signs compatible with these vertebral changes. The thoracolumbar transitional vertebrae have a large importance in the decision making of clinicians and surgeons when facing thoracic procedures. The radiologist must pay attention to this change and communicate it so that
\end{abstract}


the clinician, surgeon, and the owner can acquire knowledge, thus avoiding possible medical errors that may harm the patient.

Keywords: congenital anomaly, spine, cat, radiography

\section{Vértebra transicional toracolombar em um felino: Relato de caso}

Resumen. Vértebra transicional es una anomalía congénita caracterizada por una vertebra que adquiere características anatómicas de otra vértebra en una región adyacente y ocurre en los límites de las uniones cervicotorácico, toracolumbar, lumbosacro y sacrocoxígeo. Es la anomalía congénita más común en perros y gatos y es diagnosticado por medio de la radiografía. El presente trabajo tiene como objetivo relatar un caso de vértebra de transición toracolumbar en un felino, de 11 años, por medio de hallazgos radiográficos. La paciente fue recibida en el Laboratorio de Diagnóstico por Imagen e Cardiología (LADIC) del Hospital de Clínicas Veterinarias de la Universidad Federal de Pelotas para realizar radiografía torácica con el objetivo de investigar la presencia de metástasis pulmonares. Radiografías en proyecciones ortogonales de tórax fueron obtenidas y demostraron la presencia de costillas inseridas bilateralmente en la primera vértebra lumbar (L1), además de espondilosis a lo largo de la columna vertebral torácica. La paciente no presentaba sintomatología compatible con estas alteraciones vertebrales. La vértebra transicional toracolumbar tiene gran importancia en la tomada de decisiones de clínicos y cirujanos para procedimientos torácicos. El radiologista debe atentarse para esta alteración y comunicarla para que el clínico, cirujano y tutor tengan conocimiento, evitando de esta forma posibles errores médicos que perjudiquen al paciente.

Palabras clave: anomalía congénita, columna vertebral, gato, radiografía

\section{Introdução}

Vértebra transicional (ou vértebra de transição) é uma anomalia congênita caracterizada por uma vértebra que assume características anatômicas de outra vértebra em região adjacente e ocorre nas junções cervicotorácica, toracolombar, lombossacra (Thrall, 2019) e sacrococcígea (Morgan, 1968; Newitt et al., 2008). É uma anomalia congênita, frequentemente identificada nas radiografias de várias espécies de animais e de humanos (Flückiger et al., 2006).

A anomalia de transição mais comum na junção toracolombar em cães é a lombarização de décima terceira vértebra torácica (T13), ou seja, o desenvolvimento de processo transverso no lugar da costela de T13, podendo ser unilateral ou bilateral (Morgan, 1968). Em felinos domésticos, a anomalia de transição mais comum é na junção toracolombar, ou seja, a toracolização de primeira vértebra lombar (L1), culminando no desenvolvimento de costela unilateral ou bilateral em L1 (Newitt et al., 2008).

Estudos demonstraram que vertebras transicionais não são associadas a sinais clínicos em cães (Morgan, 1968) e gatos (Newitt et al., 2008), considerados achados incidentais, mas que podem predispor ao desenvolvimento de doença do disco intervertebral, estenose lombossacra degenerativa e displasia coxofemoral em cães (Damur-Djuric et al., 2006).

A principal importância clínica das vértebras de transição refere-se à utilização das costelas como ponto de referência para identificar locais de procedimentos cirúrgicos e clínicos, como cirurgias torácicas e toracocentese, podendo levar ao erro médico nos casos em que há mais ou menos costelas presentes (Thrall, 2019).

O presente trabalho tem como objetivo relatar um caso de vértebra de transição toracolombar em um felino, de 11 anos, abordando sobre os achados radiográficos, além de relacionar com a literatura.

\section{Relato de caso}

Foi recebida no Laboratório de Diagnóstico por Imagem e Cardiologia (LADIC) do Hospital de Clínicas Veterinárias (HCV) da Universidade Federal de Pelotas (UFPEL), uma gata, sem raça definida, com 11 anos, para realização de radiografia torácica com o objetivo de investigar a presença de 
metástase pulmonar. $\mathrm{O}$ exame foi realizado sem sedação prévia e foram obtidas radiografias em incidências ortogonais de tórax (Figura 1).
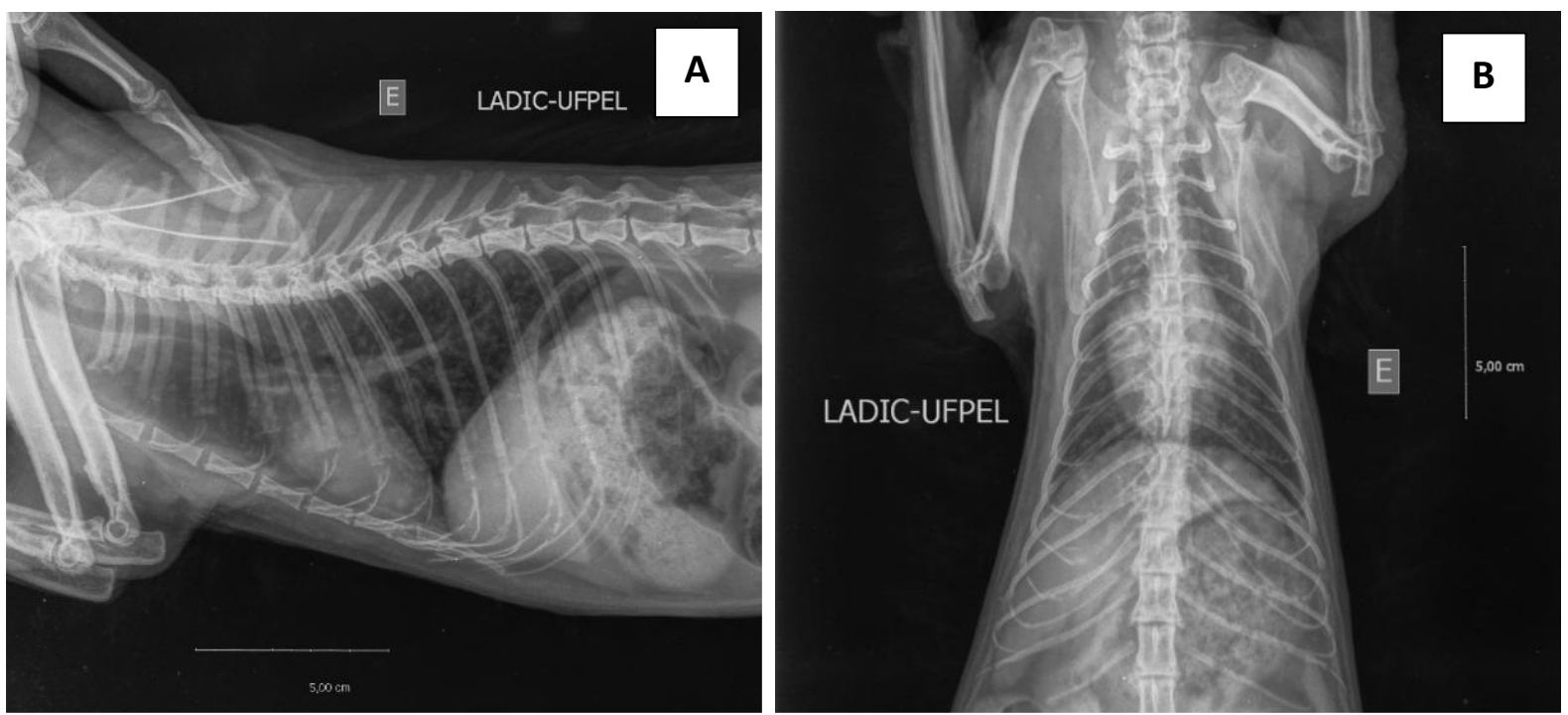

Figura 1. Imagens radiográficas torácicas evidenciando costelas inseridas bilateralmente em primeira vértebra lombar (L1). A. Projeção laterolateral esquerda (LLE). B. Projeção ventrodorsal (VD).

Nas radiografias, observaram-se costelas inseridas bilateralmente em margem cranial da primeira vértebra lombar (L1), compatível com toracolização de L1. Em associação, foram constatados entesófitos em margem cranioventral de epífise vertebral de nona (T9), décima (T10), décima primeira (T11), décima segunda (T12) e décima terceira (T13) vértebras torácicas, além de L1, compatível com espondilose deformante. Entesófitos em margem laterocaudal de corpo vertebral, bilateralmente, em vértebras T12 e T13 também foram visualizados (Figura 2).
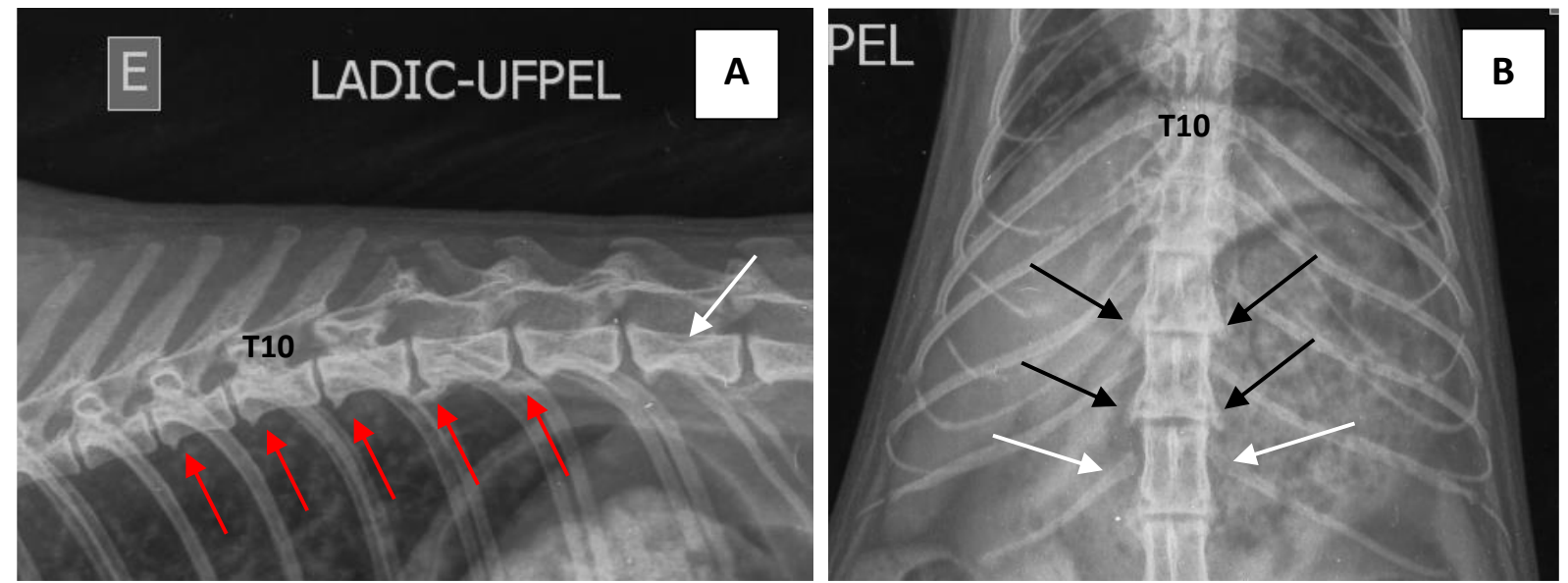

Figura 2. Imagens radiográficas aproximadas das projeções laterolateral esquerda $(\mathbf{A})$ e de ventrodorsal (B) exibindo costelas inseridas bilateralmente (setas brancas) em primeira vértebra lombar (L1), espondiloses deformantes cranioventrais nas epífises vertebrais (setas vermelhas) de nona vértebra torácica (T9) até décima terceira vértebra torácica (T13) e espondiloses deformantes caudolaterais aos corpos vertebrais (setas pretas) de décima segunda vértebra torácica (T12) e T13.

A paciente apresentou opacidade pulmonar de padrão predominantemente bronquial difuso moderado e ausência de sinais radiográficos de linfonodomegalia torácica. Não foram observadas alterações radiográficas sugestivas de metástase pulmonar. Demais estruturas musculoesqueléticas e torácicas incluídas no estudo apresentaram-se dentro da normalidade. A paciente não apresentava sinais clínicos compatíveis com as alterações encontradas. 


\section{Discussão}

As anomalias congênitas vertebrais são raras e incidentais em gatos, visto que geralmente não desenvolvem sinais clínicos (Little, 2016). Em um estudo que avaliou histologicamente alterações da medula espinhal de 205 felinos domésticos, concluiu-se que doenças inflamatórias e infecciosas foram as mais comuns $(32 \%)$, seguidas por doenças neoplásicas $(27 \%)$ e traumáticas (14\%). Não foram relatadas anomalias de transição, dado que não afetam a medula espinhal (Marioni-Henry et al., 2004). Outro estudo que avaliou radiograficamente a coluna vertebral de 200 gatos, constatou que 46 animais (23\%), apresentavam anormalidades congênitas como vértebra de transição, vértebra em bloco e vértebra ausente, sendo vértebra transicional a alteração mais comum (Newitt et al., 2008), apresentando sinônima com o presente relato.

As anomalias congênitas foram encontradas em pacientes com idade média de 6 anos, sendo vistas em gatos com 4 meses até 17 anos de idade e não possuem predisposição racial ou sexual. As vértebras transicionais sacrococcígeas e toracolombares são as mais comuns em felinos e não são associadas a sinais clínicos (Newitt et al., 2008). No presente relato, a paciente, uma felina idosa, foi recebida com outra finalidade diagnóstica e não apresentava sinais clínicos neurológicos compatíveis, o que corrobora com a literatura. Além disso, a anomalia transicional toracolombar mais comum em felinos é a toracolização de L1, que consiste na presença de costela unilateral ou bilateral em L1 em vez de processo transverso (Newitt et al., 2008).

Em relação as espondiloses deformantes observadas na paciente do presente relato, não foram encontrados estudos que correlacionem com a presença de vértebra transicional. As espondiloses são alterações degenerativas comuns em animais idosos e ocorrem devido instabilidades da coluna vertebral relacionadas a degeneração do disco intervertebral (Thrall, 2019).

Sabe-se que, em cães, vértebra transicional lombossacra (VTL) predispõe a displasia coxofemoral bilateral, estenose lombossacra degenerativa (Damur-Djuric et al., 2006) e doença do disco intervertebral (Thrall, 2019). Em gatos, não há evidências estatísticas suficientes que confirmem a relação de displasia coxofemoral e VTL (Newitt et al., 2009), porém Harris et al. (2018) sugere considerar VTL como fator predisponente para formação de estenose lombossacra degenerativa em gatos.

Não há estudos que considerem vértebra transicional toracolombar como fator predisponente para o surgimento de doenças degenerativas, como as citadas anteriormente. Contudo, segundo Newitt et al. (2008), a presença de vértebra transicional toracolombar apresenta uma grande importância clínica, já que pode levar o cirurgião a errar o local para execução de uma cirurgia com abordagem torácica, além de gerar erros na interpretação radiográfica.

\section{Conclusão}

O diagnóstico de vértebra transicional toracolombar possui grande importância clínica na tomada de decisão de clínicos e cirurgiões perante procedimentos torácicos, além de ser de extrema importância na adequada avaliação radiográfica. O radiologista deve se atentar para essa alteração e sempre comunicála para que clínico, cirurgião e tutor tomem conhecimento, evitando assim possíveis erros médicos que prejudiquem o paciente.

\section{Referências bibliográficas}

Damur-Djuric, N., Steffen, F., Hässig, M., Morgan, J. O. E. P., \& Flückiger, M. A. (2006). Lumbosacral transitional vertebrae in dogs: Classification, prevalence, and association with sacroiliac morphology. Veterinary Radiology \& Ultrasound, 47(1), 32-38. https://doi.org/10.1111/j.17408261.2005.00102.x

Flückiger, M. A., Damur-Djuric, N., Hassig, M., Morgan, J. P., \& Steffen, F. (2006). A lumbosacral transitional vertebra in the dog predisposes to cauda equina syndrome. Veterinary Radiology \& Ultrasound, 47(1), 39-44. https://doi.org/10.1111/j.1740-8261.2005.00103.x 
Harris, G., Ball, J., \& Decker, S. (2018). Lumbosacral transitional vertebrae in cats and its relationship to lumbosacral vertebral canal stenosis. Journal of Feline Medicine and Surgery, 21(4), 286-292. https://doi.org/10.1177/1098612X18774449

Little, S. E. (2016). O gato: medicina interna. In Rio de Janeiro: Roca.

Marioni-Henry, K., Vite, C. H., Newton, Ai. L., \& Winkle, T. J. (2004). Prevalence of diseases of the spinal cord of cats. Journal of Veterinary Internal Medicine, 18(6), 851-858. https://doi.org/10.1111/j.1939-1676.2004.tb02632.x

Morgan, J. P. (1968). Congenital Anomalies of the Vertebral Column of the Dog: A Study of the Incidence and Significance Based on a Radiographic and Morphologic Study. Veterinary Radiology, 9(1), 21-29. https://doi.org/10.1111/j.1740-8261.1968.tb01082.x

Newitt, A., German, A. J., \& Barr, F. J. (2008). Congenital abnormalities of the feline vertebral column. Veterinary Radiology \& Ultrasound, 49(1), 35-41. https://doi.org/10.1111/j.1740$\underline{\text { 8261.2007.00314.X }}$

Newitt, A. L. M., German, A. J., \& Barr, F. J. (2009). Lumbosacral transitional vertebrae in cats and their effects on morphology of adjacent joints. Journal of Feline Medicine and Surgery, 11(12), 941947. https://doi.org/10.1016/j.jfms.2009.01.013

Thrall, D. E. (2019). Diagnóstico de radiologia veterinária. Elsevier Editora Ltda.

Recebido: 11 de maio, 2020

Aprovado: 6 de julho, 2020.

Disponível online: 23 de outubro, 2020.
Licenciamento: Este artigo é publicado na modalidade Acesso Aberto sob a licença Creative Commons Atribuição 4.0 (CC-BY 4.0), a qual permite uso irrestrito, distribuição, reprodução em qualquer meio, desde que o autor e a fonte sejam devidamente creditados. 